\title{
Audiovestibular Manifestations in Autoimmune Disorders: A Case Clinical Control Study in Celiac Patients
}

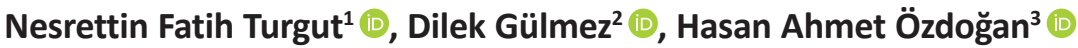 \\ ${ }^{1}$ University of Health Sciences- Samsun Health Practices and Research Center, Department of Otorhinolaryngology, Samsun, Turkey \\ ${ }^{2}$ Cerrahpasa University, Department of Audiology, Istanbul, Turkey \\ ${ }^{3}$ Cerrahpasa University, Department of Otorhinolaryngology, Istanbul, Turkey
}

ORCID ID: N.F.T. 0000-0001-6265-0058; D.G. 0000-0001-6265-0058; H.A.Ö. 0000-0003-3049-3208

Citation: Turgut NF, Gulmez D, Ozdogan HA. Audiovestibular manifestations in autoimmune disorders: a case clinical control study in celiac patients. Tr-ENT 2021;31(2):34-8. https://doi.org/10.26650/Tr-ENT.2021.951349

\begin{abstract}
Objective: The aim of this study was to evaluate audiovestibulopathy in patients with celiac disease using vestibular evoked myogenic potentials (VEMP), video head impulse test (VHIT), and audiometric examinations.

Material and Methods: Thirty-one patients with celiac disease from the gastroenterology department of Cerrahpasa Medical School, and 30 healthy controls were included in the study between 2013 and 2015. Pure tone audiometry, tympanometry, acoustic reflex test, and vestibular evaluation with VEMP and VHIT were performed in both groups. The anti-tissue transglutaminase IgA levels of all participants and disease duration of affected individuals were documented.

Results: The mean age \pm SD was $35.9 \pm 12.82(27-48)$ years and $37.6 \pm 11.6(26-48)$ years for the patient and control groups, respectively. The pure tone thresholds did not differ between the two groups. However, a subgroup of patients with high antibody levels had significantly higher highfrequency hearing thresholds. The two groups had similar VEMP test results, but a comparison of the vestibuloocular reflex (VOR) gains of six semicircular canals (SSC) revealed significant decreases in the right anterior canal in the patient group.

Conclusion: Subclinical audiovestibular pathologies can occur in patients with celiac disease. Following these patients with periodic audiovestibular test batteries may reduce patient morbidity by providing early diagnosis and rehabilitation.
\end{abstract}

Keywords: Celiac disease, VEMP, vHIT, vestibuloocular reflex

\section{INTRODUCTION}

Autoimmune diseases are known to play a role in the etiology of sensorineural hearing loss (SNHL) and vestibular dysfunction. This relationship was first reported as a separate clinical condition in 1979 by McCabe, who showed improvement in patients with bilateral progressive hearing loss using corticosteroid treatment (1). Subsequently, it was proposed that autoimmunity was the cause of inner ear pathologies, such as sudden sensorineural hearing loss or acute vertigo, because various autoimmune diseases (e.g., rheumatoid arthritis to polyartheritis nodasa, systemic lupus erythematosis, and ulcerative colitis) may include auditory and vestibular system symptoms $(2,3)$.
One of the most common autoimmune diseases is celiac disease, a food-related condition of the small intestine in humans (4). The clinical findings of celiac disease are most prominently associated with the intestinal system. However, autoimmune-related neurological system findings are also observed in $6-10 \%$ of patients. These neurological findings have been associated with autoimmunity due to the appearance of inflammatory cells in the cerebrospinal fluid, the presence of antibodies in the circulation, and the regression of symptoms after an appropriate diet (5). It has been proposed that the SNHL associated with neurological symptoms develops in patients with celiac disease due to immunological markers such as organ-unspecific autoantibodies and antineural antibodies (6). Therefore, SNHL should be defined as extraintestinal involvement in patients with celiac disease (7).

Corresponding Author: Nesrettin Fatih Turgut E-mail: drnftkbb@gmail.com

Submitted: $12.06 .2021 \bullet$ Revision Requested: 27.06.2021・ Last Revision Received: 01.07.2021・Accepted: 14.07.2021

This work is licensed under Creative Commons Attribution-NonCommercial 4.0 International License. 
The peripheral vestibular system is also a part of the inner ear and the neurological system. Therefore, we believe that this system could inevitably be affected by autoimmune mediators and that this issue should be tested. The aim of the present study was therefore to evaluate the vestibular system in patients with celiac disease, as well as their audiological systems. To the best of our knowledge, this is the first study to assess the vestibular system using VEMP and vHIT in patients with celiac disease.

\section{MATERIALS AND METHODS}

\section{Patients and study design}

The study was approved by the Istanbul University Cerrahpaşa Medical Faculty Ethics Committee (approval ID: 02/16-47473) and included 31 patients with celiac disease who were diagnosed, followed up, and treated at Cerrahpaşa Medical School Adult Gastroenterology outpatient clinic. A control group of 30 age- and sex-matched healthy volunteers was also included. None of the patients with celiac disease or the healthy volunteers had any audiovestibular complaints. Exclusion criteria included ototoxic drug use, ear surgery history, middle ear pathology, presence of spontaneous nystagmus, or presence of another autoimmune, metabolic, or neurological disease.

\section{Evaluation of serum antibody levels}

The disease activity in patients with celiac disease included in the study was determined by measuring serum antitissue transglutaminase IgA levels two weeks prior to the audiovestibular tests. The median value of $12 \mathrm{U} / \mathrm{mL}$ was used to divide the patients into a low-antibody level group (Group I) and a high-antibody level group (Group II).

\section{Audiological evaluation}

Autoscopic and routine otolaryngological examinations were performed by an expert otolaryngologist on all individuals included in the study. Acoustic impedancemetry and reflex measurements were performed using Interacoustics AZ26 and AT235H (Interacoustics A/S, Denmark) clinical tympanometry devices. Audiological evaluations were made in doublewalled IAC (Industrial Acoustic Company, Inc.) soundproof rooms in a standard quiet cabin. An Interacoustics AC 40 clinical audiometer was used to detect air conduction hearing thresholds with TDH 39P Telephonics headphones, and bone conduction hearing thresholds with a Radioear B-71 bone vibrator.

\section{Vestibular evaluation}

The VEMP test was performed with Chartr model EP 200 device (Otometrics,Natus M.,Denmark). VEMP recordings were obtained by providing a $95 \mathrm{~dB}$ tone-burst stimulus at $500 \mathrm{~Hz}$ frequency with an insert headphone. The patients were asked to contract the sternocleidomastoid muscle by turning their head to the opposite side of the stimulated ear. Amplitude and latency values were recorded by detecting $p 1$ and $\mathrm{n} 1$ waves.
The VHIT measurements were performed with an ICS impulse system (GN Otometrics, Denmark) VHIT device. During the test, eye movements were recorded with the aid of a pair of slanted glasses held to the head with rubber bands. Depending on the semicircular canal being tested, the test was performed by tilting the head right, left, front, or back by about 15 degrees and waiting for at least one second between consecutive head movements. Individuals with a semicircular canal gain score under 0.7 were considered pathological, while those scoring above 0.7 were considered normal.

\section{Statistical analyses}

SPSS 15.0 for Windows software was used for statistical analyses. Descriptive statistics were given as numbers and percentages for categorical variables and as mean and standard deviation for numerical variables. Comparison of two independent groups was made using the Student's t-test when numerical variables showed normal distribution and with the Mann-Whitney U-test when the variables did not show normal distribution. Ratios in independent groups were tested using Chi-square analysis. Correlations between numerical variables were analyzed using Pearson correlation analysis when a parametric test condition was met and with Spearman correlation analysis when the parametric test condition was not met. Statistical alpha significance level was accepted as $p<0.05$.

\section{RESULTS}

In the celiac patient group included in the study, 11 (35.5\%) were male and 20 (64.5\%) were female, with a mean age \pm of $35.9 \pm 12.82$ years ( $27-48$ years). The mean disease duration was $10.4 \pm 8.1$ years (1-29 years). Of the healthy volunteers, 14 were female $(47 \%)$ and 16 were male $(53 \%)$, with a mean age of $37.6 \pm 11.6$ years ( $26-48$ years).

The mean anti-tissue transglutaminase IgA level was $117.1 \pm 132.6 \mathrm{U} / \mathrm{mL}(0.7-300)$ in the patient group. Anti-tissue transglutaminase IgA was below $12 \mathrm{U} / \mathrm{mL}$ (Group I) in 15 (48.4\%) patients and above this value (Group II) in 16 (51.6\%) patients.

\section{Audiological findings}

No statistically significant differences were found between the patient and control group for any of the air and bone conduction frequency measurements. Air conduction thresholds in right and left ears were significantly lower, at 1000, 2000, 4000, and $6000 \mathrm{~Hz}$, and bone conduction thresholds in the right ear were also significantly lower in Group I than in Group II only at $4000 \mathrm{~Hz}$ (Table 1). Air conduction thresholds in both ears were significantly higher at all frequencies tested in the group with a disease duration of 10 or more years (Table 2).

Comparison of the gains of VHIT semicircular canals revealed a significantly higher rate of low RA (Right Anterior) in the patient group than in the control group (Table 3 ).

The results for semicircular canal gain in the vHIT test showed no significant difference between the anti-tissue transglutaminase IgA groups or different disease duration groups. 
Table 1: Comparison of the hearing thresholds $(\mathrm{dBHL})$ in the right and left ear in anti-tissue transglutaminase IgA groups of patients with celiac disease.

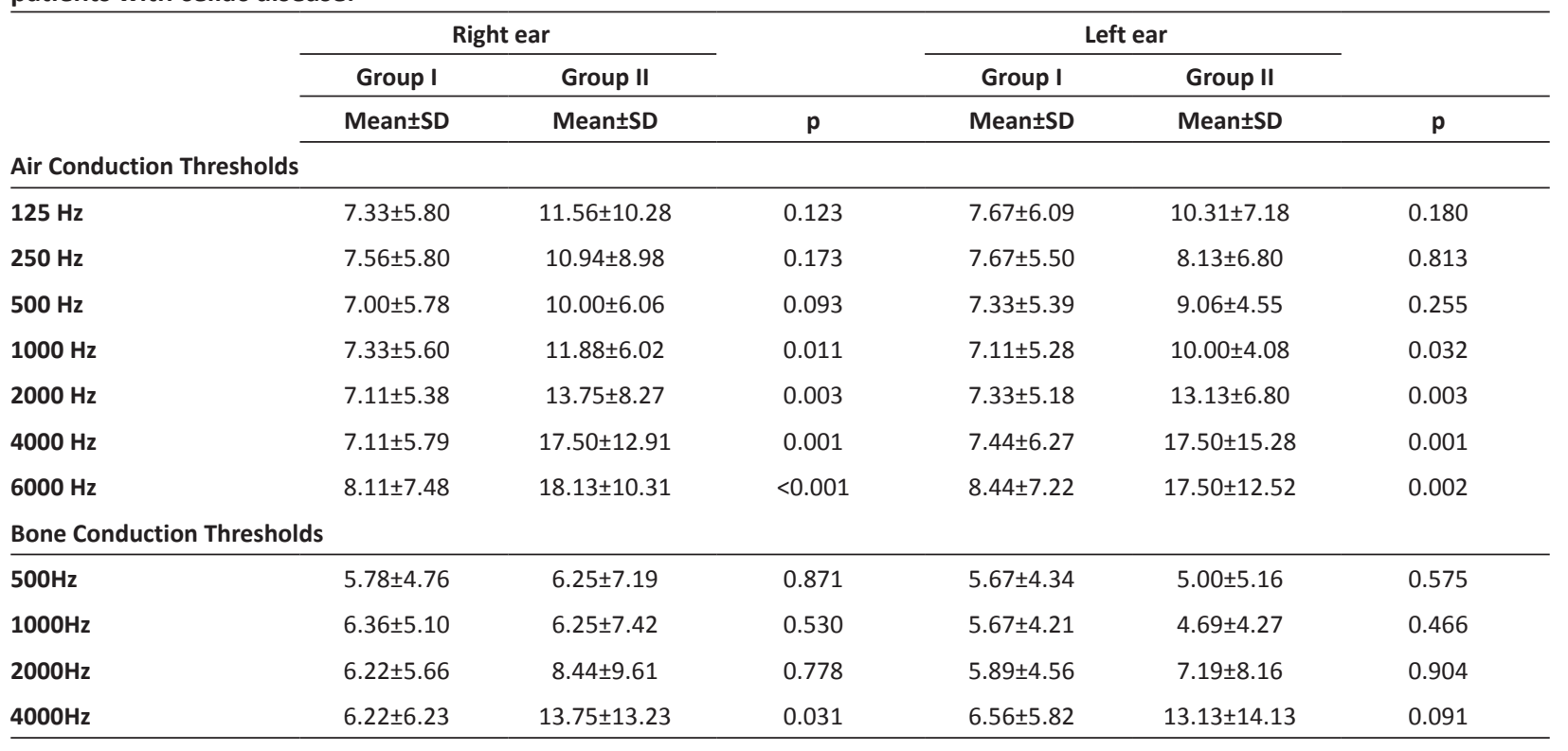

A comparison of VEMP quantitative values in patient and control groups is summarized in Table 4. No significant differences were noted between the right and left ear $\mathrm{p} 1$ and $\mathrm{n} 1$ latencies and amplitudes in the anti-tissue transglutaminase IgA groups or in the different disease duration groups.

The differences in the right and left ear $\mathrm{p} 1$ and $\mathrm{n} 1$ latency and amplitudes between patient and control groups were not statistically significant.

\section{DISCUSSION}

The pathophysiology of systemic autoimmune diseases has not yet been fully unraveled, but some publications suggest that these diseases can affect the audiovestibular system through cross-reaction, immune complex accumulation, vasculitis, or the passage of autoantibodies to the perilymph through the cerebrospinal fluid (8). The involvement of the audiovestibular system in celiac disease, an autoimmune disease, is apparently inevitable. In patients with celiac disease, the effects of the disease on the audiological system have been investigated. However, not enough attention has been paid to any potential effects on the vestibular system. To the best of our knowledge of the literature available to us, we believe that our study, with its aim of understanding the effect of celiac disease on the vestibular and audiological systems, is the first to investigate this relationship using VEMP and VHIT test batteries in this group of patients.

Autoimmune inner ear disease is clinically heterogeneous and depends on the type of immune reaction and the location of the damage to the inner ear. One study conducted in patients with psoriatic arthritis, another immune-mediated inflammatory disease, showed increases in the frequency of audiovestibular symptoms, such as hearing impairment and vertigo, and detected bilateral sensorineural hearing loss in these patients, especially at high frequencies. In addition, hearing loss prevalence was significantly higher in patients with psoriatic arthritis compared to the control group (9). Leggio et al. reported that $41.7 \%$ of patients with celiac disease had SNHL and suggested that SNHL might be considered an extraintestinal symptom of celiac disease and that immunological markers might be effective for monitoring the formation of SNHL in these patients. (10) Similarly, Umberto et al. found a higher prevalence of SNHL in patients with celiac disease than in healthy controls; however, the difference was not statistically significant (11). They also found no correlation between SNHL findings in patients with celiac disease and the presence of immunological markers (11).

In another study on pediatric patients with celiac disease, Urganci et al. found no statistically significant differences in patients in terms of mean hearing levels, disease activity and duration, or other extraintestinal symptoms (12). Conversely, Karabulut et al. performed hearing evaluations including tests of the medial olivocochlear efferent system in pediatric patients with celiac disease and found a statistically significant difference in the pure tone thresholds at $250 \mathrm{~Hz}$ frequency between the patient and control groups (13). In our study, no significant difference was found in the pure tone thresholds in both ears between the patient and control groups; however, comparison of the anti-tissue transglutaminase IgA groups revealed significantly higher pure tone thresholds at the $1000 \mathrm{~Hz}, 2000 \mathrm{~Hz}, 4000 \mathrm{~Hz}$, and $6000 \mathrm{~Hz}$ frequencies in the high antibody group than in the low antibody group. This observation of hearing impairment at high frequencies in a high antibody group might be explained by the fact that more arterial structures are present in the cochlea base than in the apex; thus, the effect of autoantibodies could be more prominent in this region. 
Table 2: Comparison of hearing thresholds $(\mathrm{dBHL})$ in the right and left ears with celiac disease duration.

\begin{tabular}{|c|c|c|c|c|c|c|}
\hline \multirow[b]{5}{*}{ Air Conduction Thresholds } & \multirow{2}{*}{\multicolumn{2}{|c|}{$\begin{array}{c}\text { Right ear } \\
\text { Disease duration }\end{array}$}} & \multirow[b]{4}{*}{$\mathbf{p}$} & & ear & \multirow[b]{4}{*}{$\mathbf{p}$} \\
\hline & & & & \multicolumn{2}{|c|}{ Disease duration } & \\
\hline & $\begin{array}{l}\text { Less than } 10 \\
\text { years }\end{array}$ & 10 years or more & & $\begin{array}{l}\text { Less than } 10 \\
\text { years }\end{array}$ & 10 years or more & \\
\hline & Mean $\pm S D$ & Mean $\pm S D$ & & Mean $\pm S D$ & Mean \pm SD & \\
\hline & & & & & & \\
\hline $125 \mathrm{~Hz}$ & $6.18 \pm 5.16$ & $13.21 \pm 10.49$ & 0.026 & $6.47 \pm 4.60$ & $12.50 \pm 8.03$ & 0.023 \\
\hline $250 \mathrm{~Hz}$ & $5.29 \pm 4.83$ & $13.93 \pm 8.13$ & 0.002 & $5.59 \pm 4.64$ & $11.07 \pm 5.94$ & 0.009 \\
\hline $500 \mathrm{~Hz}$ & $4.71 \pm 4.50$ & $12.50 \pm 5.80$ & 0.000 & $5.88 \pm 4.41$ & $11.07 \pm 4.01$ & 0.002 \\
\hline $1000 \mathrm{~Hz}$ & $6.47 \pm 4.24$ & $13.57 \pm 6.33$ & 0.002 & $6.18 \pm 4.52$ & $11.07 \pm 3.50$ & 0.003 \\
\hline $2000 \mathrm{~Hz}$ & $7.06 \pm 4.70$ & $14.29 \pm 8.96$ & 0.017 & $7.65 \pm 4.72$ & $13.57 \pm 6.91$ & 0.012 \\
\hline $4000 \mathrm{~Hz}$ & $7.65 \pm 7.52$ & $17.50 \pm 13.27$ & 0.011 & $7.06 \pm 7.51$ & $17.86 \pm 15.28$ & 0.002 \\
\hline $6000 \mathrm{~Hz}$ & $7.35 \pm 6.64$ & $19.64 \pm 10.46$ & 0.001 & $7.06 \pm 5.88$ & $20.36 \pm 11.51$ & $<0.001$ \\
\hline Bone Con & & & & & & \\
\hline $500 \mathrm{~Hz}$ & $2.94 \pm 3.56$ & $8.21 \pm 7.75$ & 0.046 & $3.82 \pm 4.52$ & $5.36 \pm 4.58$ & 0.311 \\
\hline $1000 \mathrm{~Hz}$ & $2.19 \pm 3.15$ & $9.29 \pm 6.75$ & 0.001 & $2.65 \pm 3.59$ & $6.43 \pm 3.06$ & 0.005 \\
\hline $2000 \mathrm{~Hz}$ & $2.94 \pm 3.56$ & $10.36 \pm 10.09$ & 0.039 & $4.12 \pm 3.64$ & $7.86 \pm 8.48$ & 0.293 \\
\hline $4000 \mathrm{~Hz}$ & $4.41 \pm 6.82$ & $13.93 \pm 13.61$ & 0.014 & $5.59 \pm 6.09$ & $12.86 \pm 14.77$ & 0.119 \\
\hline
\end{tabular}

Table 3: Comparison of vHIT semicircular canal gain in patients with celiac disease and healthy controls.

\begin{tabular}{lcccccc}
\hline & & \multicolumn{2}{c}{ Patient group } & \multicolumn{2}{c}{ Control group } & \\
\cline { 3 - 6 } & & $\mathbf{n}$ & $\%$ & $\mathbf{n}$ & $\%$ & $\mathbf{p}$ \\
\cline { 3 - 6 } RA & Normal & 20 & 64.5 & 27 & 90.0 & 0.018 \\
& Low & 11 & 35.5 & 3 & 10.0 & \\
\multirow{2}{*}{ LA } & Normal & 26 & 83.9 & 30 & 100 & 0.053 \\
& Low & 5 & 16.1 & 0 & 0.0 & \\
\multirow{2}{*}{ RP } & Normal & 31 & 100.0 & 27 & 90.0 & 0.113 \\
& Low & 0 & 0.0 & 3 & 10.0 & \\
\multirow{2}{*}{ LP } & Normal & 30 & 96.8 & 28 & 96.6 & 1.000 \\
& Low & 1 & 3.2 & 1 & 3.4 & \\
\multirow{2}{*}{ RL } & Normal & 29 & 96.7 & 28 & 93.3 & 1.000 \\
& Low & 1 & 3.3 & 2 & 6.7 & \\
\multirow{2}{*}{ LL } & Normal & 29 & 100 & 30 & 100 & - \\
\hline
\end{tabular}

RA: right anterior; LA: left anterior; RP: right posterior; LP: left posterior; RL: right lateral; LL: left lateral

An important point to note is that autoimmune diseases cause inner ear involvement through autoimmune mediators, and this situation will affect the vestibular system in addition to the hearing system. In their immune-mediated inner ear disease review, Bovo et al. concluded that the vestibular system was affected in $50 \%$ of autoimmune inner ear patients, and that these vestibular system symptoms include imbalance, ataxia, positional vertigo, and episodic vertigo (8).
Table 4: Comparison of VEMP quantitative values in patients with celiac disease and healthy controls.

\begin{tabular}{|c|c|c|c|}
\hline & Patient group & Control group & p \\
\hline & Mean $\pm S D$ & Mean $\pm S D$ & \\
\hline Right p1 latency & $14.16 \pm 2.01$ & $14.82 \pm 2.67$ & 0.658 \\
\hline Right n1 latency & $20.80 \pm 3.43$ & $21.03 \pm 1.86$ & 0.744 \\
\hline $\begin{array}{l}\text { Right p1 } \\
\text { amplitude }\end{array}$ & $32.82 \pm 3.30$ & $34.66 \pm 4.94$ & 0.093 \\
\hline $\begin{array}{l}\text { Right n1 } \\
\text { amplitude }\end{array}$ & $43.29 \pm 3.70$ & $44.68 \pm 5.72$ & 0.064 \\
\hline Left p1 latency & $14.03 \pm 2.67$ & $14.59 \pm 2.48$ & 0.649 \\
\hline Left n1 latency & $21.00 \pm 3.81$ & $20.61 \pm 2.48$ & 0.640 \\
\hline Left $p 1$ amplitude & $34.44 \pm 3.37$ & $35.62 \pm 5.17$ & 0.650 \\
\hline Left $n 1$ amplitude & $43.46 \pm 3.30$ & $44.90 \pm 5.76$ & 0.051 \\
\hline
\end{tabular}

In a case study presenting Behçet's disease manifesting with peripheral vestibulopathy, Alison et al. reported that a 66-yearold male patient being followed up with hearing and vestibular system complaints was diagnosed with Behçet's disease as mouth and genital ulcers developed approximately ten years after the initial complaints (14). An evaluation of the vestibular system of this patient using ice-water caloric stimulation revealed permanent right canal paralysis and low amplitude response in both ears. His VHIT test revealed catch-up saccades in right side rotation, fewer saccades in left side rotation, and a significant decrease in VOR gain, while his VEMP test showed a lack of right ear stimulation, suggesting weakness in the right otolith organs. In that case study, the authors emphasized that 
patients who were referred to a clinic with peripheral vestibular system pathologies and SNHL should be evaluated in detail and investigated for the presence of an underlying autoimmune disease (14).

Nahid et al. used VEMP to evaluate the vestibular system in patients with rheumatoid arthritis, another commonly observed autoimmune disease (15). The CVEMP measurements of 25 rheumatoid arthritis patients and 20 healthy controls with a stimulus intensity of $95 \mathrm{dBnHL}$ at $500 \mathrm{~Hz}$ turn-burst stimulation revealed no significant difference in absolute or interaural amplitudes between the two groups. However, prolonged latency was detected in the patient group. Prolonged latency is an abnormal characteristic finding of central vestibulopathy and has been reported to indicate vestibulospinal path lesions; therefore, the authors suggested that vestibular rehabilitation may be useful in early diagnosis.

Pawlak-Oniskia et al. evaluated the vestibular system in a pediatric celiac patient group using ENG and VEMP test batteries but found no significant difference in VEMP results between the patients and healthy controls (16). However, the ENG results frequently indicated gaze nystagmus, disordered eye-tracking, and optokinetic nystagmus, and the authors argued that these findings support the hypothesis that neurological findings may be observed early in patients with celiac disease. In our study, we evaluated the vestibular system in patients with celiac disease using VHIT and VEMP test batteries. The VEMP test results did not differ significantly between the patient and control groups. However, the vHIT results revealed a greater frequency of low right anterior semicircular canal gain in the celiac patient group. This observation could be a finding of subclinical vestibular pathology; therefore, we believe it is important that these patients be followed up for vestibular pathologies as well.

The small sample size in the present study and the use of VHIT and VEMP test batteries alone for vestibular evaluation are the most significant limitations of this study. Therefore, we suggest that a need exists for the evaluation of the vestibular systems of large groups of patients with celiac disease using additional test batteries.

\section{CONCLUSION}

The effects of systemic autoimmune diseases on the audiovestibular system necessitate the screening of patients with appropriate test batteries for early diagnosis, as this will enable early detection of potential inner ear damage. Audiovestibular rehabilitation can then prevent the increase in morbidity in these patients. Our data showed that subclinical audiovestibular pathologies may occur in patients with celiac disease, another autoimmune disease. We believe that periodic follow-up of these patients with audiovestibular test batteries may reduce patient morbidity by providing patients with early diagnosis and timely implementation of rehabilitation programs.

Ethics Committee Approval: The study was approved by the Istanbul University Cerrahpaşa Medical Faculty Ethics Committee (approval ID: 02/16-47473).
Informed Consent: Written informed consent was obtained from all patients included in the study after detailed information about the study was given.

Peer-Review: Externally peer-reviewed.

Author Contributions: Conception/Design of Study- N.F.T., D.G.; Data Acquisition- N.F.T., D.G.; Data Analysis/Interpretation- N.F.T.; Drafting Manuscript- N.F.T., D.G.; Critical Revision of Manuscript- A.H.Ö.; Final Approval and Accountability- N.F.T., A.H.Ö.

Conflict of Interest: Authors declared no conflict of interest.

Financial Disclosure: Authors declared no financial support.

\section{REFERENCES}

1. McCabe BF. Autoimmune sensorineural hearing loss. Ann Otol Rhinol Laryngol 1979;88:585-9.

2. Harris J, Ryan A. Fundamental immune mechanisms of the brain and inner ear. Otolaryngol - Head Neck Surg 1995;112:639-53.

3. Öztürk A, Yalçin Ş, Kaygusuz I, Şahin S, Gök Ü, Karlidağ T, Ardıçoğlu Ö. High-frequency hearing loss and middle ear involvement in rheumatoid arthritis. Am J Otolaryngol 2004;25:411-7.

4. Farrell RJ, Kelly CP. Diagnosis of celiac sprue. Am J Gastroenterol 2001;96:3237-46.

5. Briani C, Zara G, Alaedini A, Grassivaro F, Ruggero S, Toffanin E, et al. Neurological complications of celiac disease and autoimmune mechanisms: a prospective study. J Neuroimmunol 2008;195:171-5.

6. Alaedini A, Green PH. Narrative review: celiac disease: understanding a complex autoimmune disorder. Ann Intern Med 2005;142:289-98.

7. Green PH, Jabri B. Celiac disease. Annu Rev Med 2006;57:207-21.

8. Bovo R, Aimoni C, Martini A. Immune-mediated inner ear disease. Acta Otolaryngol 2006;126:1012-21.

9. Amor-Dorado JC, Barreira-Fernandez MP, Pina T, Vázquez-Rodríguez TR, Llorca J, González-Gay MA. Investigations into audiovestibular manifestations in patients with psoriatic arthritis. J Rheumatol 2014;41:2018-26.

10. Leggio L, Cadoni G, D’Angelo C, Mirijello A, Scipione S, Ferrulli A, et al. Coeliac disease and hearing loss: preliminary data on a new possible association. Scand J Gastroenterol 2007;42:1209-13.

11. Volta U, Ferri GG, De Giorgio R, Fabbri A, Parisi C, Sciajno L, et al. Sensorineural hearing loss and celiac disease: a coincidental finding. Can J Gastroenterol 2009;23:531-5.

12. Urganci $N$, Kalyoncu $D$, Calis $A B$. Sensorineural hearing loss in pediatric patients with celiac disease. Eur Arch Otorhinolaryngol 2015;272:2149-51.

13. Karabulut H, Hizli S, Dagli M, Karabulut I, Acar B, Celik E, et al. Audiological findings in celiac disease. ORL J Otorhinolaryngol Relat Spec 2011;73:82-7.

14. White AS, Taylor RL, McNeill C, Garsia R, Welgampola MS. Behçet's disease presenting as a peripheral vestibulopathy. J Clin Neurosci 2014;21:1060-3.

15. Heydari N, Hajiabolhassan F, Fatahi J, Movaseghi S, Jalaie S. Vestibular function can be affected by autoimmune processes in rheumatoid arthritis. Aud Vest Res 2015;24:193-200.

16. P Pawlak-Osińska K, Kaźmierczak H, Kuczyńska R, SzaflarskaPopławska. Looking for the auditory and vestibular pathology in celiac disease [in Polish]. Otolaryngol Pol 2007;61:178-83. 